\title{
Resuscitation of Patient with Suspected/Confirmed COVID-19: How to Increase Medical Staff Safety
}

\author{
(ㄱ) Semih Korkut ${ }^{1}$, (1) Togay Evrin² , (1) Burak Katipoğlu², (1) Jacek Smereka ${ }^{3}$, (1) Anna Drozd ${ }^{4}$, (1) Lukasz Szarpak \\ 1Department of Emergency Medicine, Kartal Dr. Lütfi Kırdar Training and Research Hospital, University of Health Sciences Turkey, İstanbul, Turkey \\ ${ }^{2}$ Clinic of Emergency Medicine, Dr. Rıdvan Ege Training and Research Hospital, Ufuk University Faculty of Medicine, Ankara, Turkey \\ ${ }^{3}$ Department of Emergency Medical Service, Wroclaw Medical University, Wroclaw, Poland \\ ${ }^{4}$ Polish Society of Disaster Medicine, Warsaw, Poland \\ ${ }^{5}$ Bialystok Oncology Center, Bialystok, Poland
}

\section{Dear Editor,}

Cardiopulmonary resuscitation (CPR) is a substantial burden for medical personnel, particularly during the 2019 Novel Coronavirus (COVID-19) pandemic. Although the precise transmission mechanisms of the new coronavirus are presently unclear, human-to-human transmission can occur, and the risk of airborne spread during aerosol-generating medical procedures remains a concern in certain circumstances (1).

Medical personnel, including physicians, nurses, and paramedics, as provided by the Center for Disease Control and Prevention, should be in full personal protective equipment (PPE) during aerosol-generating procedures (2,3). CPR exemplifies an aerosolgenerating procedure. Notably, as the mortality rate in COVID-19 is $7 \%$ because of the occurrence of viral pneumonia and progressive hypoxia occurring in COVID-19, it can be concluded that resuscitation is a frequent and essential lifesaving procedure. In the resuscitation room, patients in the most severe condition are treated; hence a variety of emergency medical equipment is available. Medical personnel of the resuscitation room is emergency physicians, nurses, and paramedics experienced in treating critically severe patients.

Due to the risk of infectious aerosol, a key component of resuscitation should be endotracheal intubation and assisted breathing through a respirator; this fully supports the respiratory system, simultaneously reducing the risk of contamination. Difficulties consequential to wearing a face mask, goggles, protective visor, and double gloves appoint video-laryngoscopy as the preferred method of intubation in comparison to direct laryngoscopy. Non-invasive ventilation for the treatment of COVID-19 patients should be used as early as possible, and in some cases, it may ensure that endotracheal intubation is avoided; however, in the case of resuscitation, endotracheal intubation is the standard procedure.

The use of PPE can increase the discomfort of the rescuer; besides, it hinders the performance of medical procedures, including getting intravascular access. As indicated by research, therefore, the intramedullary injection should be recommended (4), which concerning drug pharmacokinetics and pharmacodynamics, is in no way inferior to peripheral vascular access. Some patients under emergency procedures require rapid sequence intubation with intravenous anesthetics, opioids, and muscle relaxants. The need for endotracheal intubation by the most experienced medical personnel and the use of videolaryngoscopy should be noted, as in severe initial hypoxia, increased hypoxia due to prolonged endotracheal intubation or insertion of the tube into the esophagus may have fatal consequences.

Another important element of resuscitation is high-quality chest compressions. During CPR, however, performing chest compression may cause the rescuer's face protection devices to adhere to exposing the rescuer to viral infection (5) poorly. To prevent such a situation, it is reasonable to use automated chest compression devices (ACCDs) during the resuscitation of COVID-19 patients. Though the European Resuscitation Council guidelines do not recommend routine ACCDs, it should be noted

Cite this article as: Korkut S, Togay E, Katipoğlu B, Smereka J, Drozd A, Szarpak L. Resuscitation of Patient with Suspected/ 
that the current extraordinary situation COVID-19 narrates the use of extraordinary solutions such as ACCDs. Application ACCDS beyond chest compressions allows for transport of patients to the hospital while carrying out chest compression with minimal risk of infection to the rescuer.

In summary, it is imperative to exercise extreme caution in aerosol-generating procedures during the COVID-19 pandemic. Furthermore, to reduce the risk of infection of medical personnel, resuscitation should include endotracheal intubation, intramedullary punctures, and ACCDs.

Keywords: Cardiopulmonary resuscitation, COVID-19, SARSCoV-2, endotracheal intubation, intraosseous access, chest compression, personal protective equipment, safety

\section{Ethics}

Peer-review: Externally and internally peer-reviewed.

\section{Authorship Contributions}

Concept: T.E., L.S., Design: T.E., B.K., A.D., Data Collection or Processing: S.K., T.E., L.S., Analysis or Interpretation: T.E., A.D.,
LS., Literature Search: S.K., B.K., J.S., Writing: S.K., T.E., B.K., J.S., A.D., L.S.

Conflict of Interest: No conflict of interest was declared by the authors.

Financial Disclosure: The authors declared that this study received no financial support.

\section{References}

1. Dzieciatkowski T, Szarpak L, Filipiak KJ, Jaguszewski M, Ladny JR, Smereka J. et al. COVID-19 challenge for modern medicine. Cardiol J. 2020;27:175-83.

2. Ruetzler K, Szarpak L, Filipiak KF, Ladny JR, Smereka J. The COVID-19 pandemic - a view of the current state of the problem. Disaster Emerg Med J. 2020;5:106-7.

3. Smereka J, Szarpak L. The use of personal protective equipment in the COVID-19 pandemic era. Am J Emerg Med. 2020;38:1529-30.

4. Smereka J, Szarpak L, Filipiak KJ, Jaguszewski M, Ladny JR. Which intravascular access should we use in patients with suspected/confirmed COVID-19? Resuscitation. 2020;151:8-9.

5. Hwang SY, Yoon H, Yoon A, Kim T, Lee G, Jung KY, et al. N95 filtering facepiece respirators do not reliably afford respiratory protection during chest compression: A simulation study. Am J Emerg Med. 2020;38:12-7. 